\title{
COVID-19 highlights risks of healthcare and social care workers attending work while ill
}

Sally Hall Dykgraaf, Jane Desborough, Catherine Kelaher, Michael Kidd

AS CoVID-19 affects healthcare and social care systems around the world, reports of infection among healthcare and social care workers (HSCWs) continue to accumulate. These include outbreaks in hospitals, primary care, aged care, disability care, community and home care services, and draw attention to the risks of HSCWs contracting COVID-19 and also subsequently acting as vectors for transmission. Reducing this possibility is a key component of reducing the impact of SARS-CoV-2. ${ }^{1}$

The risks of occupational acquisition of COVID-19 when employees attend work with SARS-CoV-2 infection have been highlighted. ${ }^{2}$ This behaviour raises particular problems for healthcare facilities and settings. In Australia at the time of writing (22 May 2020), where community transmission rates for COVID-19 are relatively low, several major outbreaks relate to possible cases of HSCW-mediated transmission. ${ }^{3,4}$ In the following discussion we consider HSCWs, in alignment with the national Work Health and Safety Act 2011, ${ }^{5}$ to be any person who works as part of a healthcare or social care service, practice or business. This includes employees, contractors, sub-contractors, students, volunteers, administrative staff and clinicians.

While extensive literature explores the productivity and performance implications of attending work while unwell (presenteeism), workers with an infectious illness also pose a public health hazard, which is amplified in healthcare and other care settings because of the presence of vulnerable populations. ${ }^{6}$ Rates of infectious illness presenteeism (IIP) reportedly range from $35 \%$ to $97 \%$ of workers and are higher among doctors and nurses than for workers in other professions. ${ }^{7}$ While discussions of IIP generally refer to workers with relevant symptoms, in the COVID-19 context, workers who are infected but asymptomatic may also contribute to transmission risk.

Studies of influenza transmission suggest that while many workers are compliant with sick leave recommendations, substantial transmission occurs in the workplace. A recent cohort study of HSCWs with laboratory-confirmed influenza found $14.1 \%$ continued to work while ill, ${ }^{8}$ and previous studies have shown HSCWs felt pressure to work while unwell with influenza-like illness, despite being aware of the risks to patients and colleagues. ${ }^{9}$

In a pandemic, the isolation of infectious cases required to mitigate transmission presents challenges for those without access to paid sick leave (PSL). PSL can increase the likelihood of workers staying at home with infectious illnesses and reduce rates of IIP, especially in settings where financial considerations might compel staff to continue working or return to work earlier than is desirable. In modelling studies, the availability of PSL and addition of extra 'flu leave' days have acted to substantially modify workplace transmission rates. ${ }^{10}$

While PSL is important, other factors also affect HSCW attendance at work when unwell. These include assessments of one's own health, economic and lifestyle stresses, the work environment, contract conditions, professional culture and individual characteristics. ${ }^{79}$ Both job demands and job security are particularly important influences in shaping presenteeism behaviour. These factors may combine in ways that simultaneously weaken health and wellbeing but drive motivation to continue working. ${ }^{11}$

Structural and system elements can also function as antecedents for IIP. For example, in a fee-for-service payment environment, health professionals in private practice may constitute a high-risk group. In addition to personal, professional or collegial disincentives to be absent from work, if the health professional is a practice owner, they may have difficulty ensuring business continuity during absences as a result of continuing financial overheads.

IIP is a multidimensional problem. Explanations are often layered and context specific - and vary between different types of HSCWs. ${ }^{12}$ For example, medical personnel describe difficulties finding replacement staff and concern for the impact of absence on patients and colleagues, as well as sociocultural norms related to the perception that taking sick leave is a weakness. ${ }^{9}$ HSCWs at long-term care facilities where PSL is not universal describe the inability to afford lost pay. ${ }^{13}$

Most pandemic plans emphasise workforce surge capacity and screening, testing and protection of HSCWs. However, few consider the individual, organisational, workplace and system barriers that may contribute to IIP-related risks of HSCW-mediated transmission. Pandemic planning and response strategies would benefit from 
incorporating approaches to resolving behavioural responses such as IIP, which have the potential to increase disease transmission in healthcare and social care settings. At the facility level, organisational culture should be reviewed to assess specific weaknesses that may increase the likelihood of IIP. The adoption of measures that acknowledge the complex causes underlying IIP - such as additional sick leave, PSL for casual workers, targeted education and training, and enhanced job security - will assist in supporting HSCW decisions to stay at home when unwell during a pandemic.

\section{First published online 4 June 2020.}

\section{Authors}

Sally Hall Dykgraaf RN, Grad Cert ClinMan, PhD Scholar, Action Research, COVID-19 Primary Care Response Group (seconded), Primary Care Division, Australian Government Department of Health, ACT; Research Manager, Rural Clinical School, Australian National University, ACT. sally.hall@anu.edu.au Jane Desborough RN, RM, MPH, PhD, Action Research, COVID-19 Primary Care Response Group (seconded), Primary Care Division, Australian Government Department of Health, ACT; Senior Research Fellow, Research School of Population Health, College of Health and Medicine, Australian National University, ACT; Program Leader, Health Experience Team, Our Health in Our Hands $(\mathrm{OHIOH})$, Australian National University, ACT

Catherine Kelaher MBBS (Hons), FAFOEM, FRACGP, GradDipOccEnvMed, Principal Medical Advisor, Office of Health Protection, Australian Government Department of Health, ACT

Michael Kidd AM, MD, FAHMS, Deputy Chief Medical Officer and Principal Medical Advisor, Primary Care Division, Australian Government Department of Health, ACT; Professor of Primary Care Reform, Australian National University, ACT; Adjunct Professor, Department of Family and Community Medicine, University of Toronto, Canada; Emeritus Director, World Health Organization Collaborating Centre on Family Medicine and Primary Care, GVA; Professorial Fellow, Murdoch Children's Research Institute, The Royal Children's Hospital Melbourne, Vic; Honorary Professor of Global Primary Care, Southgate Institute for Health, Society and Equity, Flinders University, SA

Competing interests: This work received in-kind support from the Australian Government Department of Health and the Australian National University, where the authors are employed.

Provenance and peer review: Not commissioned, peer reviewed.

Citation: Hall Dykgraaf S, Desborough J, Kelaher C, Kidd M. COVID-19 highlights risks of healthcare and social care workers attending work while ill. Aust J Gen Pract 2020:49 Suppl 23. doi: 10.31128/AJGPCOVID-23.

\section{References}

1. Heneghan C, Oke J, Jefferson T. COVID-19 how many healthcare workers are infected? CEBM. 17 April 2020. Available at www.cebm.net/covid-19/ covid-19-how-many-healthcare-workers-areinfected [Accessed 3 June 2020].

2. Eisen D. Employee presenteeism and occupational acquisition of COVID-19. Med J Aust 2020.

3. Nguyen K, Raper A. Cornavirus concerns at western Sydney aged-care facility where woman worked six shifts before testing positive. ABC News. 14 April 2020. Available at www.abc.net. au/news/2020-04-14/resident-son-angry-afteranglicare-worker-contracts-coronavirus/12144862 [Accessed 3 June 2020].

4. ABC News. Coronavirus alert for nursing homes in Tasmanian outbreak zone. ABC News. 16 April 2020. Available at www.abc.net.au/ news/2020-04-16/coronavirus-alert-for-nursinghomes-in-tasmanian-outbreak-zone/12154884 [Accessed 3 June 2020].

5. Federal Register of Legislation. Work health and safety act 2011. No. 137, 2011. Compilation No. 9. Forrest, ACT: Office of Parliamentary Counsel, 2011. Available at www.legislation.gov.au/Details/ C2018C00293 [Accessed 3 June 2020].

6. Widera E, Chang A, Chen HL. Presenteeism: A public health hazard. J Gen Intern Med 2010;25(11):1244-47. doi: 10.1007/s11606-0101422-x.

7. Webster RK, Liu R, Karimullina K, Hall I, Amlôt R, Rubin GJ. A systematic review of infectious illness presenteeism: Prevalence, reasons and risk factors. BMC Public Health 2019;19(1):799. doi: 10.1186/s12889-019-7138-x.

8. Imai C, Hall L, Lambert SB, Merollini KMD. Presenteeism among health care workers with laboratory-confirmed influenza infection: A retrospective cohort study in Queensland, Australia. Am J Infect Control 2020;48(4):355-60. doi: 10.1016/j.ajic.2019.07.024.

9. Szymczak JE, Smathers S, Hoegg C, Klieger S, Coffin SE, Sammons JS. Reasons why physicians and advanced practice clinicians work while sick: A mixed-methods analysis. JAMA Pediatr 2015;169(9):815-21. doi: 10.1001/ jamapediatrics.2015.0684.

10. Kumar S, Grefenstette JJ, Galloway D, Albert SM, Burke DS. Policies to reduce influenza in the workplace: Impact assessments using an agent-based model. Am J Public Health 2013;103(8):1406-11. doi: 10.2105/ AJPH.2013.301269.

11. Miraglia M, Johns G. Going to work ill: A metaanalysis of the correlates of presenteeism and a dual path model. J Occup Health Psychol 2016;21(3):261-83. doi: 10.1037/ocp0000015.

12. Giæver F, Lohmann-Lafrenz S, Løvseth LT. Why hospital physicians attend work while ill? The spiralling effect of positive and negative factors. BMC Health Serv Res 2016;16(1):548. doi: 10.1186/ s12913-016-1802-y.

13. Chiu S, Black CL, Yue $X$, et al. Working with influenza-like illness: Presenteeism among US health care personnel during the 2014-2015 influenza season. Am J Infect Control 2017;45(11):1254-58. doi: 10.1016/j. ajic.2017.04.008. 